\title{
INTERAÇÃO MEDICAMENTOSA ENTRE FÁRMACOS ANTI-HIPERTENSIVOS EM USUÁRIOS DE BEBIDA ALCOÓLICA
}

\section{ANTIHYPERTENSIVE DRUGS INTERACTION AMONG USERS OF ALCOHOLIC BEVERAGES}

Lídia Cíntia De Jesus Silva*, Maria Emilia Santos Pereira Ramos**

Autora para correspondência: Lídia Cíntia De Jesus Silva - lidiacintia@gmail.com

*Enfermeira Especialista em Cardiologia e Hemodinâmica pela Escola Bahiana de Medicina e Saúde Pública

**Doutora em Farmacologia pela Universidade Federal do Ceará - UFC. Professora na Universidade Estadual de Feira

de Santana e na Escola Bahiana de Medicina e Saúde Pública

R E S U M O

\begin{abstract}
A Hipertensão Arterial Sistêmica (HAS) é considerada uma condição clínica de importante problema para a saúde pública mundial. Apesar da eficácia medicamentosa ser comprovada, o comportamento do indivíduo com relação à mudança no estilo de vida também contribui significativamente para prevenir o desenvolvimento da HAS. Dessa forma, objetivou-se investigar a relação entre fármacos anti-hipertensivos em usuários de bebidas alcoólicas, e implementar um modelo de esclarecimento a população classificada como hipertensos. Trata-se de um estudo de pesquisa bibliográfica integrativa, onde foi utilizado um total de 48 publicações. A pesquisa revelou que o consumo concomitante de fármacos anti-hipertensivos e bebidas alcoólicas eleva os níveis de pressão arterial provavelmente por utilizarem a mesma via metabólica (citocromo P450). E grande parte da população desconhece a gravidade dessa doença (evolução lenta e assintomática e possibilidade de morte). Conclui-se que profissionais de saúde, em especial o enfermeiro, possui papel fundamental no esclarecimento dos riscos dessa associação.
\end{abstract}

Palavras-chave: Consumo de Bebidas Alcoólicas; Hipertensão; Enfermagem; Anti-Hipertensivos; Adesão à Medicação. 
Systemic arterial hypertension (SAH) is considered a clinical condition and major concern for global public health. Despite the drug efficacy being proven, the individual's behavior with respect to the change in lifestyle also contributes significantly to prevent the development of hypertension. This work aimed to investigate the relationship between antihypertensive drugs in alcohol users, and to implement means of educationto of the population classified as hypertensive. It is an integrative literature review study, which employed a total of 48 publications. The survey showed that the concomitant use of antihypertensive drugs and alcohol raises blood pressure levels because both substances use the same metabolic pathway (cytochrome P450). And much of the population is unaware of the severity of the disease (asymptomatic and slow evolution and possible death). It was concluded that health professionals, especially nurses, have a primary role in clarifying the risks of this association.

Key Words: Alcohol Drinking; Hypertension; Nursing; Antihypertensive Agents; Medication Adherence. 


\section{INTRODUÇÃO}

A Hipertensão Arterial Sistêmica (HAS) é uma patologia crônica multifatorial em que há elevação do nível de pressão arterial e apesar de possuir fatores de risco modificáveis é uma condição clínica de importante problema para a saúde pública mundial, porque traz consequências a longo prazo'. Caracterizada como um fenômeno associado a um risco elevado de mortalidade por doenças cardiovasculares e cerebrovasculares (provoca incapacidades funcionais, alterações estruturais dos órgãos-alvo e alterações metabólicas) reduzindo assim qualidade de vida ${ }^{1-2}$.

Sabe-se que a hipertensão arterial sistêmica é também considerada um importante fator de risco por não apresentar sintomatologia, em parte da população acometida, e por levar a eventos cardiovasculares fatais e não fatais, destacandose assim, o acidente vascular encefálico (AVE) e a doença isquêmica do coração (DIC $)^{3-5}$.

Cerca de 7,6 milhões de mortes no mundo são atribuíveis à elevação da pressão arterial. Nos Estados Unidos essa doença é altamente prevalente sendo que um em cada três adultos americanos possui a probabilidade de desenvolver hipertensão(6). De acordo com a VI Diretrizes Brasileira de Hipertensão, a hipertensão no Brasil apresenta uma prevalência média de $32,5 \%$ na população adulta atingindo até $75 \%$ após os 70 anos.

Em função da alta prevalência de HAS no mundo, é comum encontrar pacientes que utilizam fármacos anti-hipertensivos como medida de controle da doença. Essa terapia é utilizada para prevenir doenças cardiovasculares e manter os níveis de pressão arterial controlados abaixo de $130 \times 85 \mathrm{mmHg}^{1-7}$. Na regulação da pressão arterial além das medidas farmacológicas, também são recomendadas medidas primárias como redução do peso, controle do açúcar e alimentos gordurosos, exercício físico e diminuição do consumo de bebidas alcoólicas $^{1-8}$. Apesar da eficácia medicamentosa ser comprovada, o comportamento do indivíduo com relação à mudança no estilo de vida também contribui significativamente para prevenir 0 desenvolvimento de $\mathrm{HAS}^{1,5,9}$.

consumo mundial de bebidas alcoólicas é uma conduta legal, bem como, um comportamento aceito na maioria das culturas e é altamente prevalente sendo responsável cerca de $3,2 \%$ de todas as mortes que acontecem no mundo. Dos consumidores mundiais, aproximadamente 2 bilhões de pessoas consomem bebidas alcoólicas. Em 2012, ficou constatado que cerca de $51,5 \%$ dos adultos norteamericanos consumiram bebidas alcoólicas e dados semelhantes foram descritos por Laranjeiras ${ }^{10}$ no Brasil, em que cerca de $52 \%$ dos adultos acima dos 18 anos consumiam bebida alcoólica.

Segundo Klatsky ${ }^{11}$ o etanol quando consumido de moderado a grandes quantidades pode provocar alterações morfológicas em praticamente todos os órgãos e tecido do corpo, principalmente $\circ$ fígado, o coração e o estômago. Quando utilizado concomitantemente com medicamentos pode levar a diversas consequências, como a redução pela metade da meia vida biológica do fármaco, podendo potencializar seu efeito e induzir o antagonismo farmacológico. Esses efeitos podem ocorrer até mesmo quando o consumo é realizado em baixas concentrações. Essa associação é mostrada independentemente da idade adulta, do sexo, do grupo racial e étnico e dos demais fatores de risco para hipertensão (ingestão de sódio, alimentos ricos em gordura, tabagismo, etc. $)^{11-12}$.

Seu consumo é considerado um fator de risco controlável e evitável, segundo Wenshu ${ }^{13}$ seu uso por períodos prolongados pode aumentar a pressão arterial contribuindo fortemente para $\circ$ desenvolvimento da hipertensão e consequentemente altos índices de mortalidade por doenças cardiovasculares, acidente vascular cerebral e síndrome metabólica ${ }^{1,14-18}$. Atualmente tem-se considerado que o consumo de etanol acima de $30 \mathrm{~g} /$ dia, equivalente a $210 \mathrm{~g} / \mathrm{semana}$, está associado há um aumento significativo da pressão arterial sistólica e diastólica e o risco de desenvolver hipertensão ${ }^{19}$. Foi encontrado também, a quantidade de etanol por dose padrão, a qual demonstra que ao beber 1 lata de cerveja ou 1 copo de chopp, 1 taça de vinho e 1 dose de destilada (uísque, pinga, vodka), cujos teores alcoólicos correspondem respectivamente $5 \%, 14 \%$ e $50 \%$, ingere-se cerca de $12-14 \mathrm{~g}$ de álcool puro (etanol) atingindo níveis de $0,2 \mathrm{~g}$ de álcool por litro de sangue ${ }^{20}$.

Sendo assim, é muito comum encontrar indivíduos que 
interrompem o tratamento medicamentoso anti-hipertensivo para consumir bebida alcoólica em alguma ocasião ou os utilizam simultaneamente (combinando a medicação com o etanol), percebe-se que essas relações de consumo medicamento/álcool tem recebido pouca atenção por parte do poder público em divulgar os prováveis malefícios e pela comunidade científica em pesquisar as possíveis interações do álcool com medicamentos antihipertensivos" . Dessa forma o presente estudo sucinta a seguinte questão investigativa: "Qual a relação entre fármacos anti-hipertensivos em usuários de bebida alcoólica e como implementar um modelo de esclarecimento a população classificada como hipertensos? e possui como objetivo investigar a relação entre fármacos antihipertensivos em usuários de bebidas alcoólicas, e implementar um modelo de esclarecimento a população classificada como hipertensos.

\section{MÉTODOS}

Trata-se de um estudo de pesquisa bibliográfica integrativa baseado em publicações científicas em inglês, espanhol e português, no período compreendido entre 1915 e 2013 . Foram utilizados como descritores e suas combinações: Consumo de Bebidas Alcoólicas, Hipertensão, Enfermagem, Anti-Hipertensivos, Adesão à Medicação. Foram selecionados um total de 48 publicações (Quadro 1), que atendiam os critérios de inclusão. As bases de dados consultadas foram PubMed, Medline e
Portal Capes e livros de Farmacologia Básica. Foram incluídos apenas artigos que abordaram o consumo de bebidas alcoólicas associado a fármacos antihipertensivos e suas complicações. Excluindo aquele que relacionavam o consumo de álcool a outras doenças.

A análise dos artigos incluídos nesta revisão foi iniciada com vistas a identificar a temática central abordada no estudo. A partir desta análise, três categorias foram construídas para uma melhor elaboração da síntese dos conteúdos.

Quadro 1. Estudos incluídos na revisão integrativa

\begin{tabular}{|c|c|c|}
\hline ANO & AUTOR & TÍTULO \\
\hline 1915 & Lian C. & L'alcoolisme cause d'hypertension artérielle. \\
\hline 1977 & $\begin{array}{c}\text { Klastsky AL, Friedmann GD, Siegelaub AB, } \\
\text { Gerard MJ. }\end{array}$ & Alcohol consumption and blood pressure. \\
\hline 1992 & Sikkink, J, Fleming, $M$. & Health effects of alcohol. \\
\hline 2000 & $\begin{array}{l}\text { Krauss RM, Eckel RH, Appel IJ, Daniels SR, } \\
\text { Deckelbaum RJ, Erdman JW, et al. }\end{array}$ & $\begin{array}{l}\text { AHA Dietary - Guidelines. A Statement for } \\
\text { healthcare professionals from de nutrition } \\
\text { Committee of the American Heart Association. }\end{array}$ \\
\hline 2001 & $\begin{array}{l}\text { Fuchs FD, Chambless LE, Whelton PK, Nieto FJ, } \\
\text { Heiss G. }\end{array}$ & $\begin{array}{l}\text { Alcohol Consumption and the Incidence of } \\
\text { Hypertension: The Atherosclerosis Risk in } \\
\text { Communities Study. }\end{array}$ \\
\hline 2003 & Jackson VA, Sesso WD, Buring JE, Gaziano JM. & $\begin{array}{l}\text { Alcohol Comsumption and Mortality in Men With } \\
\text { Preexisting Cerebrovascular Disease. }\end{array}$ \\
\hline 2004 & $\begin{array}{c}\text { Renaud SC, Gueguen R, Conard P, Lanzmann } \\
\text { PD, Orgogozo JM, Henry O. }\end{array}$ & $\begin{array}{l}\text { Moderate wine drinkers have lower hypertension- } \\
\text { related mortality: a prospective cohort study in } \\
\text { French men. }\end{array}$ \\
\hline 2004 & $\begin{array}{c}\text { Reims HM, Kieldsen SE, Brady WE, Dahlo B, } \\
\text { Devereux RB, Julius S, et al. }\end{array}$ & $\begin{array}{l}\text { Alcohol consumption and cardiovascular risk in } \\
\text { hypertensives with left ventricular hypertrophy: the } \\
\text { LIFE study. }\end{array}$ \\
\hline 2004 & $\begin{array}{c}\text { Stranges S, Wu T, Dorn JM, Freudenheim JL, } \\
\text { Multi P, Farinaro E, et al. }\end{array}$ & $\begin{array}{l}\text { Relationship of alcohol drinking pattern to risk of } \\
\text { hypertension: a population-based study. }\end{array}$ \\
\hline 2005 & Huntgeburth M, Freyhaus HT, Rosenkranz S. & Alcohol Consumption and Hypertension. \\
\hline 2005 & Rehm J, Monteiro M. & $\begin{array}{l}\text { Alcohol consumption and burden of disease in the } \\
\text { Americas: implications for alcohol policy. }\end{array}$ \\
\hline 2005 & Morales-Olivas FJ, Estan L. & Antihypertensive drug-drug interactions. \\
\hline 2005 & Steffens, AA. Fuchs FD. Moreira LB. & $\begin{array}{l}\text { Incidência de hipertensão arterial pelo consume } \\
\text { de álcool: é modificável pela raça? [Dissertação]. }\end{array}$ \\
\hline
\end{tabular}




\begin{tabular}{|c|c|c|}
\hline ANO & AUTOR & TÍTULO \\
\hline 2006 & $\begin{array}{c}\text { Klatsky AL, Koplik S, Gunderson E, Kipp H, } \\
\text { Friedman GDI. }\end{array}$ & $\begin{array}{c}\text { Sequelae of Systemic Hypertension in } \\
\text { Alcohol Abstainers, Light Drinkers, and Heavy } \\
\text { Drinkers. }\end{array}$ \\
\hline 2006 & Goodman \& Gilman. & Bases Farmacológicas da terapêutica. \\
\hline 2006 & Sociedade Brasileira de Cardiologia. & $\begin{array}{l}\text { V Diretrizes Brasileiras de Hipertensão. } \\
\text { Arquivo Brasileiro de Cardiologia. }\end{array}$ \\
\hline 2006 & $\begin{array}{c}\text { Lessa I, Magalhães L, Araúio MJ, Filho NA, } \\
\text { Aquino E, Mônica MC. }\end{array}$ & $\begin{array}{c}\text { Arterial Hypertension in the Adult Population } \\
\text { of Salvador (BA). }\end{array}$ \\
\hline 2007 & Laranjeiras R. et al. & $\begin{array}{c}\text { I Levantamento Nacional sobre os padrões } \\
\text { de consumo de álcool na população } \\
\text { brasileira. }\end{array}$ \\
\hline 2007 & Baynes JW, Dominiczak MH. & Bioquímica médica. \\
\hline 2007 & Guimarães S, Moura D, Silva P. & $\begin{array}{l}\text { Terapêutica Medicamentosa e as suas Bases } \\
\text { Farmacológicas. }\end{array}$ \\
\hline 2008 & Klatsky AL, Gunderson E. & Alcohol and hypertension: a review. \\
\hline 2008 & Wakabayashi I. & $\begin{array}{c}\text { Relationships among alcohol drinking, blood } \\
\text { pressure and serum cholesterol in healthy } \\
\text { young women. }\end{array}$ \\
\hline 2008 & Pereira RE. & $\begin{array}{c}\text { Relação entre o consume de bebidas } \\
\text { alcoólicas e as infrações de trânsito } \\
\text { [Dissertação]. }\end{array}$ \\
\hline 2008 & Chen L, Smith GD, Harbord RM, Lewis SJ. & $\begin{array}{l}\text { Alcohol and blood pressure: A systematic } \\
\text { review implementing a mendelian } \\
\text { randomization approach. }\end{array}$ \\
\hline 2008 & $\begin{array}{c}\text { Sesso HD, Cook NR, Buring JE, Manson JE, } \\
\text { Gaziano JM. }\end{array}$ & $\begin{array}{l}\text { Alcohol Consumption and the Risk of } \\
\text { Hypertension in Women and Men. }\end{array}$ \\
\hline 2008 & Bibi Z. & $\begin{array}{l}\text { Retracted: Role of cytochrome P450 in drug } \\
\text { interactions. }\end{array}$ \\
\hline 2009 & $\begin{array}{l}\text { Núnez Córdoba JM, Martínez-González MA, } \\
\text { Bes-Rastrollo M, Toledo E, Beunza JJ, Alonso A. }\end{array}$ & $\begin{array}{l}\text { Alcohol Consumption and the Incidence of } \\
\text { Hypertension in a Mediterranean Cohort: The } \\
\text { SUN Study. }\end{array}$ \\
\hline 2009 & $\begin{array}{c}\text { Wilsnack RW, Wilsnack SC, Kristianson AF, } \\
\text { Vogeltanz- Holm ND, Gmel G. }\end{array}$ & $\begin{array}{c}\text { Alcohol Consumption: Patterns From The } \\
\text { Multinational Genacis Project. }\end{array}$ \\
\hline 2009 & $\begin{array}{l}\text { Lloyd-Jones D, Adams R, Carnethon M, Simone } \\
\text { G, Ferguson TB, Flegal Ket al. }\end{array}$ & $\begin{array}{c}\text { Doença cardíaca e derrame estatísticas } \\
\text { - atualização: um relatório do Comité da } \\
\text { American Heart Association Statistics and } \\
\text { Stroke Subcomissão. }\end{array}$ \\
\hline 2010 & $\begin{array}{c}\text { Bos S, Grobbee DE, Boer JMA,Verschuren } \\
\text { WM, Beulens JWJ. }\end{array}$ & $\begin{array}{c}\text { Alcohol Consumption and Risk of } \\
\text { Cardiovascular Disease Among Hypertensive } \\
\text { Women. }\end{array}$ \\
\hline 2010 & Sociedade Brasileira de Hipertensão. & $\begin{array}{c}\text { VI Diretrizes da Sociedade Brasileira de } \\
\text { Hipertensão. }\end{array}$ \\
\hline 2010 & $\begin{array}{l}\text { Halanych JH, Safford MM, Kertesz SG, Plet- } \\
\text { cher MJ, Kim YI, Person SD, et al. }\end{array}$ & $\begin{array}{c}\text { Alcohol consumption in young adults and } \\
\text { incident hypertension: } 20 \text {-year follow-up } \\
\text { from the coronary artery risk development in } \\
\text { young adults study. }\end{array}$ \\
\hline 2010 & Klatsky AL. & Alcohol and cardiovascular health. \\
\hline
\end{tabular}




\begin{tabular}{|c|c|c|}
\hline ANO & AUTOR & TíTULO \\
\hline 2010 & Wakabayaski I. & $\begin{array}{c}\text { History of Antihypertensive Therapy Influences } \\
\text { the Relationships of Alcohol With Blood Pressure } \\
\text { and Pulse Pressure in Older Men. }\end{array}$ \\
\hline 2010 & Silva MEDC. Moura MEB. & $\begin{array}{l}\text { Representações sociais da hipertensão } \\
\text { arterial elaboradas por portadoras e } \\
\text { profissionais de saúde: uma contribuição da } \\
\text { enfermagem. [Dissertação- Mestrado]. }\end{array}$ \\
\hline 2010 & Maciel KF. Mantovani MF. Mazza VA. & $\begin{array}{l}\text { Rede de apoio social de portadores de } \\
\text { hipertensão arterial para o cuidado. } \\
\text { [Dissertação- Mestrado]. }\end{array}$ \\
\hline 2010 & Ungari AQ, Fabbro ALD. & $\begin{array}{c}\text { Adherence to drug treatment in hypertensive } \\
\text { patients on the Family Health Program. }\end{array}$ \\
\hline 2012 & Garrido BJ, Lobera J. & $\begin{array}{l}\text { Interactions between antihypertensive } \\
\text { drugs and food. As interações entre anti- } \\
\text { hipertensivos e alimentos }\end{array}$ \\
\hline 2012 & Frederico PM. & $\begin{array}{l}\text { Interações medicamentosas potenciais dos } \\
\text { anti-hipertensivos: uso perigoso entre idosos } \\
\text { [Dissertação]. }\end{array}$ \\
\hline 2012 & $\begin{array}{c}\text { Roger VL, Go AS, Lloyd-Jones DM, Benjamin } \\
\text { EJ, Berry JD, Borden WB, et al. }\end{array}$ & Heart disease and strok statistics. \\
\hline 2012 & $\begin{array}{c}\text { Wang } H \text {, Wang J, Liu } M M \text {, Wang } D \text {, Liu } Y Q \text {, } \\
\text { Zhao } Y \text {, et al. }\end{array}$ & $\begin{array}{l}\text { Epidemiology of general obesity, abdominal } \\
\text { obesity and related risk factors in urban } \\
\text { adults from } 33 \text { communities of northeast } \\
\text { china: the CHPSNE study. }\end{array}$ \\
\hline 2012 & $\begin{array}{c}\text { Arranz S, Chiva-Blanch G, Valderas-Martínez } \\
\text { P, Medina-Remón A, Lamuela-Raventós RM, } \\
\text { Estruch R. }\end{array}$ & $\begin{array}{l}\text { Wine, Beer, Alcohol and Polyphenols on } \\
\text { Cardiovascular Disease and Cancer. }\end{array}$ \\
\hline 2013 & $\begin{array}{c}\text { Simino J, Sung YJ, Kume R, Schwander K, Rao } \\
\text { DC. }\end{array}$ & $\begin{array}{c}\text { Gene-alcohol interactions identify several } \\
\text { novel blood pressure loci including a } \\
\text { promising locus near. }\end{array}$ \\
\hline 2013 & $\begin{array}{l}\text { Pajak A, Szafraniec K, Kubinova R, Malyutina } \\
\text { S, Peasey A, Pikhart H, et al. }\end{array}$ & $\begin{array}{l}\text { Binge Drinking and Blood Pressure: Cross- } \\
\text { Sectional Results of the HAPIEE Study. }\end{array}$ \\
\hline 2013 & Akhmedjonov A, Suvankulov F. & $\begin{array}{l}\text { Alcohol consumption and its impact on the } \\
\text { risk of high blood pressure in Russia. }\end{array}$ \\
\hline 2013 & $\begin{array}{l}\text { Valencia-Martín JL, Galán I, Guallar-Castillón } \\
\text { P, Rodríguez-Artalejo F. }\end{array}$ & $\begin{array}{c}\text { Alcohol drinking patterns and health related } \\
\text { quality of life reported in the spainsh. }\end{array}$ \\
\hline 2013 & $\begin{array}{c}\text { Luo W, Guo Z, Hao C, Yao X, Zhou Z, Wu M. } \\
\text { et al. }\end{array}$ & $\begin{array}{l}\text { Interaction of current alcohol consumption } \\
\text { and abdominal obesity on hypertension risk. }\end{array}$ \\
\hline 2013 & Brasil. Ministério da saúde. & $\begin{array}{c}\text { Departamento de Atenção Básica. } \\
\text { Estratégias Para o Cuidado da Pessoa } \\
\text { Com Doença Crônica. Hipertensão Arterial } \\
\text { Sistêmica. }\end{array}$ \\
\hline
\end{tabular}

\section{Consumo de álcool x Hipertensão Arterial Sistêmica (HAS)}

\section{A associação entre álcool e hipertensão arterial} sistêmica foi descrita primeiramente em 1915 por Lian $^{21}$ apud Steffens ${ }^{22}$, mas somente em 1977 Klatsky et al. ${ }^{23}$ observaram essa associação em uma grande população.

A terapêutica combinada dos anti-hipertensivos com o etanol pode causar diversas consequências para o organismo, e além disso, afeta a eficácia da terapia anti-hipertensiva. 
Os fármacos anti-hipertensivos são escolhidos de acordo com o grau de risco cardiovascular, com a avaliação da presença de comorbidade e/ou de acordo com os estágios de classificação da pressão arterial. A depender do estágio da pressão arterial, os anti-hipertensivos podem até ser usados de forma combinada com dois ou mais anti-hipertensivos.

A classe de fármacos disponíveis no Brasil para - tratamento medicamentoso da hipertensão são diuréticos (hidroclorotiazida, furosemida, espirolactona), betabloqueadores seletivos (Atenolol, succinato de metoprolol e tartarato de metropolol), betabloqueadores não seletivos (propranolol), agentes alfa e betabloqueadores (caverdilol), antiadrenérgicos de ação central (metildopa), bloqueadores seletivos dos canais de cálcio (Bensilato de anlodipino, nifedipino, cloridrato de verapramil), os vasodilatadores diretos (cloridrato de hidralazina), inibidores da enzima conversora de angiotensina (captopril, meleato de enalapril, losartana potássica). Vários estudos relatam que - uso clínico dessas classes medicamentosa, de 3 a 4 anos demostraram redução de morbidade e mortalidade cardiovascular ${ }^{1-24}$.

Sabe-se que os fármacos anti-hipertensivos têm a finalidade de não só reduzir a pressão arterial, mas também evitar complicações cardiovasculares fatais e não fatais, reduzindo assim a taxa de mortalidade ${ }^{1-25}$. Porém, apesar desses benefícios, quando utilizados em conjunto com outras substâncias, como o álcool, pode levar danos à saúde.

O fígado possui um papel central no metabolismo do indivíduo por apresentar uma variedade de funções bioquímicas; ele recebe sangue venoso do intestino, e assim, todos os produtos da digestão, além dos fármacos e substâncias, passam por ele antes de entrar na circulação sistêmica; o fígado ainda possui a capacidade de desintoxicar uma grande variedade de xenobióticos e também possui sua função excretora ${ }^{26}$.

A maioria das fármacos é metabolizada no fígado. O metabolismo hepático aumenta a solubilidade das drogas, consequentemente sua excreção. Quando duas substâncias são ingeridas concomitantemente e usam a mesma via de biotransformação pode ocorrer entre elas uma interação ${ }^{26-27}$.
O etanol é oxidado no fígado, principalmente, pelo álcool desidrogenase, para formar acetaldeído, que por sua vez é oxidado pelo aldeído desidrogenase (ALDH) em acetato, e a maior parte deste atinge outras partes do organismo pela corrente sanguínea onde participa de outros ciclos metabólicos. Essas etapas são realizadas através das Fases I e II catalisada pelos citocromo $\mathrm{P} 450^{28}$. Semelhantemente $\circ$ metabolismo dos fármacos anti-hipertensivos ocorre pela mesma via, utilizando a oxidação da fase l, mediada pela atividade enzimática do citocromo P450 (CYP450) encontrada principalmente em hepatócitos. A síntese hepática, nos citocromos P450 é aumentada por certas substâncias em um processo conhecido como 'indução' que aumenta a velocidade das reações da fase I resultando em perda da eficácia de outros fármacos. Inversamente, as drogas que formam um complexo relativamente estável com uma das enzimas do citocromo P450 em particular, inibem - metabolismo de outros fármacos, pois reduz a atividade enzimática e retarda a formação de metabólitos, consequentemente um aumento da concentração de fármaco (substrato) disponível para a produção do efeito, o que pode levar à toxicidade ${ }^{26-30}$.

Quando o etanol é ingerido, $90 \%$ é absorvido pelo organismo e sua distribuição começa rápida depois lentifica, sendo eliminado apenas $5 \%$ através da urina. Atualmente considerado indutor enzimático, aumenta a atividade do sistema de enzima e a taxa de formação de metabolito, reduzindo a concentração de fármacos no organismo em condições de atuar, o que pode levar a perda de efeito terapêutico. Uma das hipóteses resulta desse papel indutor do etanol quando consumido cronicamente, esse efeito tem sido observado quando ocorre concomitantemente - uso de fármacos antihipertensivos e consumo de bebidas alcoólicas, possibilitando diminuição da concentração farmacológica e resultando em aumento da pressão arterial $^{26-29}$.

Outras hipóteses sugerem aumento dos níveis do $\mathrm{Ca}+$ intracelular com aumento subsequente da reatividade vascular, estimulando o endotélio a liberar endotelina e inibindo a produção de NO (óxido nítrico) dependente do endotélio, resultando em aumento da pressão arterial. Ou que o álcool apresenta propriedades vasopressora direta por mecanismos desconhecidos ${ }^{26-29}$. 
Foram relatados por Guimarães e colaborados $^{31} \mathrm{e}$ Klatsky ${ }^{11}$ que o uso de doses agudas de etanol não possuem, via de regra geral, ações intensas sobre a função cardíaca, porém embora raro, podem ocorrer arritmias ventriculares e supraventriculares, sobretudo a fibrilação auricular, devido à ingestão de quantidades elevadas de bebidas alcoólicas. Além de que a carência de tiamina (obtida através da alimentação) pode ser uma das causas de insuficiência cardíaca, já que a mesma está reduzida nos usuários crônicos de etanol. Relatam ainda que estudos recentes têm concluído que a prevalência de alcoólicos crônicos em populações de hipertensos é superior à de populações com valores tensionais normais.

Contrariamente têm sido descritos efeitos benéficos do consumo moderado de álcool em estudos que abordam a doença cardíaca coronária e doenças arteriovasculares, descrevendo que os efeitos do etanol a nível vascular dependem das doses e da frequência do seu consumo. Estudos epidemiológicos realizados na França sugeriram que o consumo de vinho (20 a 30g/dia) seria um dos fatores que confeririam um efeito cardioprotetor na população que consomem alta ingestão dietética de gorduras saturadas e que apresentavam níveis séricos elevados de colesterol, reduzindo de 10 a $40 \%$ - risco de desenvolver cardiopatia coronariana quando comparadas aos indivíduos que não consumiam bebidas alcoólicas ${ }^{32}$. Apesar das evidencias que o consumo moderado de álcool é muito menos prejudicial e até benéfico, a inexistência de um limite rígido para todas as pessoas, ou seja, não existe estanquicidade naquilo que se pode considerar altas e baixas doses de álcool para cada pessoa, limita sua utilidade benéfica a saúde ${ }^{11}$. Apesar dessas evidências, quando se avalia a associação do etanol e hipertensão arterial, as elevações pressóricas têm sido observadas mesmo em consumo leve de etanol ${ }^{33}$. Levando ao aumento da incidência de insuficiência cardiovascular que inclui arritmias, miocardiopatia e acidente vascular encefálico hemorrágico, que acabam por suplantar os efeitos benéficos do álcool nas artérias ${ }^{34}$.

\section{Complicações da interação entre o consumo de bebida alcoólica e fármacos anti-hipertensivos}

As principais complicações entre $\circ$ consumo de bebidas alcoólicas e fármacos anti-hipertensivos são observadas entre indivíduos que apresentam um padrão de consumo conhecido mundialmente como "binge drink", ou seja, o consumo de grandes quantidades de álcool ou ainda, consumo de um volume excessivo ${ }^{10,34,35,36}$. Do total da população adulta brasileira, $28 \%$ já beberam em "binge", ou seja, consumiram bebida alcoólica de forma abusiva pelo menos 1 vez no ano ${ }^{10}$.

Essa relação causal entre o consumo de álcool e níveis elevados de pressão arterial (PA) foi reforçada por estudos desenvolvidos por Stranges et al. ${ }^{37}$ e Renaud et al. ${ }^{38}$ que observaram aumento da PA a partir do consumo diário de $30 \mathrm{~g}$ de álcool, mas discordantes em relação ao consumo moderado. Porém segundo Huntgeburth ${ }^{39}$, Klatsky ${ }^{11}$, Akhmedjonov ${ }^{40}$ indivíduos normotensos que consumem $1 \mathrm{~g} /$ dia de álcool já é observado um aumento permanente da pressão arterial sistólica (PAS) em 0,24 mmHg, assim como o consumo de $10 \mathrm{~g} /$ dia de etanol aumenta a PAS em 1 a $2 \mathrm{~mm} \mathrm{Hg}$. Observações essas confirmadas em numerosos estudos que demonstram que o aumento da PAS acontece também em indivíduos hipertensos e acontece independente de outros fatores de risco da HAS, como idade, sexo, raça, peso corporal e ingestão de sal. Diminuir ou restringir o consumo de álcool leva a uma redução da PA e um aumento significativo da potência e eficácia terapêutica anti-hipertensiva. Fato comprovado em estudos de intervenção de redução de consumo de álcool onde observou-se diminuição da pressão arterial e novamente elevação da mesma com a reintrodução do consumo de etanol, demonstrando assim, a reversibilidade dos efeitos do álcool na elevação da PA $^{11,12,33,39,41,42}$.

Segundo Krauss ${ }^{43}$, a redução do consumo de etanol para a maioria dos indivíduos deve ser de $30 \mathrm{~g}$ de álcool/dia, valor encontrado em $600 \mathrm{~mL}$ de cerveja ( $5 \%$ de álcool), $250 \mathrm{~mL}$ de vinho ( $12 \%$ de álcool) e $60 \mathrm{~mL}$ de destilados (uísque, vodca, aguardente com $50 \%$ de teor alcoólico), porém para indivíduos com sobrepeso e/ou triglicérides elevados, homens de baixo peso e mulheres, esse limite deve ser reduzido à metade. A redução ou o abandono do consumo de bebidas alcoólicas pode reduzir de 2 a $4 \mathrm{mmHg} \mathrm{na}$ pressão arterial.

Estudos têm demonstrado que o consumo de etanol 
possui forte relação de grande impacto sobre a prevalência de hipertensão e ainda, duplica o risco de desenvolver patologias cardíacas coronarianas como cardiomiopatia alcoólica, distúrbios do ritmo cardíaco (arritmia cardíaca - fibrilação atrial) ${ }^{25}$. O consumo de um volume excessivo de etanol, independente do gênero, afeta os órgãos vitais do corpo tais como: cérebro, fígado, coração e afeta também o sistema imunológico. No cérebro leva a alterações no humor e no comportamento do indivíduo, no fígado pode levar a inflamações e alterações no seu funcionamento, causando problemas como: cirrose hepática, esteatose, fibrose e até mesmo hepatite; no sistema imunológico, o indivíduo fica mais propenso a contrair doenças como pneumonia e tuberculose, pois diminui a capacidade do corpo para evitar infecções. Nos consumidores de grandes quantidades de álcool foram também observados efeitos periféricos e centrais, dentre eles um aumento para $\circ$ risco de hemorragia subaracnóide e menor risco de acidente vascular cerebral oclusivo ${ }^{11,33,39,44}$.

\section{Adesão ao tratamento farmacológico da hipertensão dos consumidores de bebida alcoólica}

Observa-se também que, $\circ$ aumento da pressão arterial pelos consumidores de bebidas alcoólicas reside na falta de adesão ao tratamento medicamentoso da $\mathrm{HAS}^{9-45}$ principalmente nos dias de maior consumo de álcool, pois parte dessa população acredita que 0 uso concomitante pode trazer prejuízos à saúde, o que acaba agravando os quadros de hipertensão arterial. Apesar dos avanços tecnológicos no campo da produção dos medicamentos, sua crescente utilização pelas populações e as limitações de conhecimento a respeito da segurança dos fármacos, trouxeram também reconhecimento de que os medicamentos além de ajudar no restabelecimento da saúde, podem causar danos, principalmente quando utilizados juntamente com bebidas alcoólicas.

Tanto no tratamento medicamentoso como no tratamento não farmacológico é papel dos profissionais de saúde, especialmente o profissional de enfermagem, o esclarecimento à população sobre a hipertensão arterial e suas possíveis complicações. Quanto maior conhecimento sobre a doença maiores chances de se reduzir a morbidade e mortalidade por doenças cardiovasculares ${ }^{46}$.

Portanto, é indispensável que $\circ$ profissional de enfermagem alerte a este público sobre a importância de aderir ao tratamento medicamentoso e deve ainda, motivar o indivíduo no processo de mudanças deste hábito, desta forma, proporcionará uma melhor efetividade terapêutica ${ }^{24}$.

Ao articular o conhecimento entre a população, - profissional de saúde passa a compreender as dificuldades enfrentadas pelos portadores da HAS e seus familiares, quanto ao uso adequado da medicação e consumo concomitante de bebidas alcoólica. Segundo Silva ${ }^{47}$ a maior parte da população desconhece a gravidade dessa doença, que inclui seu curso clínico, cronicidade, evolução lenta e assintomática, possibilidade de morte. Embora seja uma doença de grande agravo à saúde, muitas pessoas não utilizam a terapêutica de modo adequado nem modificam estilo de vida, que inclui abolir o uso de bebidas alcoólicas.

Apesar de todas as complicações decorrentes do consumo do álcool nos pacientes hipertensos, é raro a quantidade de estudos que relacionam o consumo de bebidas alcoólicas e a qualidade de vida da população. Muito embora a hipertensão possua seu caráter crônico, graças aos grandes avanços tecnológicos na assistência à saúde foi possível aumentar a expectativa de vida da população. Dessa forma, é necessário incentivar mudanças no modo e no estilo de vida, provocando rupturas em hábitos deletérios diários. Ao contrário do que tem sido relatado, os estudos de Valencia-Martín et al. ${ }^{19}$ identificaram uma melhor qualidade de vida física nos indivíduos que fazem grande consumo de bebida alcoólica do que os abstêmios, porém chamam a atenção para as limitações do estudo, ainda assim, esses dados não deveriam ser utilizados para estímulo ao consumo de bebidas alcoólicas.

O profissional de enfermagem, como membro da equipe multiprofissional de saúde pode desenvolver estratégias de educação em saúde através de palestras sobre $\circ$ uso de anti-hipertensivos $e$ bebidas alcoólicas e suas consequências, realizar um acompanhamento mais de perto (consultas semanais) da hipertensão dos pacientes que declaram consumir 
bebidas alcoólicas e estimular a busca por ajuda para abandono do vício do álcool através dos CAPS (Centro de Atenção Psicossocial - Álcool e Droga), possibilitando melhor enfrentamento da doença. Segundo Maciel $^{48}$, o profissional envolvido nesse processo também deve envolver a família, pois, considera-a como uma importante fonte de apoio que poderá contribuir no cuidado, assim como, o auxiliando no enfrentamento das dificuldades.

O enfermeiro deve acompanhar este usuário com verificação semanal da pressão arterial nas consultas de enfermagem que são ofertados pelos serviços de Unidade de Saúde da Família (USF) com orientações sobre hábitos de vida saudável, uso de etanol, sobre o uso correto da medicação, importância da adesão ao tratamento e a continuidade de monitorização da pressão arterial. Desta forma, o enfermeiro poderá avaliar se a pressão arterial está controlada, se o indivíduo aderiu ao tratamento recomendado, avaliar também se o medicamento está sendo eficaz à aquele indivíduo e além disso avaliar a motivação do indivíduo para o tratamento e a sua capacidade de auto-cuidado. De acordo com esta avaliação, o enfermeiro também pode encaminhar este paciente para o apoio do Núcleo de Apoio à Saúde da Família (NASF) que contém outros profissionais de saúde de diferentes áreas, ajudará na superação de sua condição ${ }^{24}$.

\section{CONCLUSÃO}

Baseado nos estudos é possível afirmar que em indivíduos hipertensos, a depender do padrão de consumo, o etanol é um importante fator de risco para aumento dos níveis pressóricos e complicações cardiovasculares. Sendo assim, os profissionais de saúde, em especial o enfermeiro, têm um papel fundamental no esclarecimento dos riscos dessa associação, assim como, estabelecer estratégias educacionais e de vigilância a essa população, proporcionando redução de danos à saúde.

\section{REFERÊNCIAS}

1. Sociedade Brasileira de Hipertensão. VI Diretrizes Brasileiras de Hipertensão. 2010;13(1):34-46

2. Roger VL, Go AS, Lloyd-Jones DM, Benjamin EJ, Berry JD, Borden WB et al. Heart disease and strok statistics - 2012 update: a report from the American Heart Association. Circulation. 2012;125(1):e2-e220. doi: 10.1161/ CIR.0b013e31823ac046

3. Klatsky AL, Koplik S, Gunderson E, Kipp H, Friedman GD. Sequelae of Systemic Hypertension in Alcohol Abstainers, Light Drinkers, and Heavy Drinkers. The American Journal of Cardiology. 2006;98(8):1063-68. doi: 10.1016/i. amjcard.2006.05.029

4. Chen L, Smith GD, Harbord RM, Lewis SJ. Alcohol and blood pressure: A systematic review implementing a mendelian randomization approach. PLoS Medicine. 2008;5(3):e52. doi: 10.1371 /journal.pmed.0050052

5. Lessa I, Magalhães L, Araújo MJ, Filho NA, Aquino $E$, Mônica MC. Arterial Hypertension in the Adult Population of Salvador (BA) - Brazil. Arquivos Brasileiros Cardiologia. 2006;87(6):68392. doi: 10.1590/S0066-782X2006001900011

6. Lloyd-Jones D, Adams R, Carnethon M, Simone $G$, Ferguson TB, Flegal $K$ et al. Doença cardíaca e derrame estatísticas - 2009 atualização: um relatório do Comité da American Heart Association Statistics and Stroke Subcomissão. Circulation. 2009;1 19(3):480-6. doi: 10.1161/ CIRCULATIONAHA.108.191259

7. Sociedade Brasileira de Cardiologia. V Diretrizes Brasileiras de Hipertensão. Arquivo Brasileiro de Cardiologia. 2006.1-48.

8. Wakabayashi I. History of Antihypertensive Therapy Influences the Relationships of Alcohol With Blood Pressure and Pulse Pressure in Older Men. American Journal of hypertension. 2010;23(6):633-38. doi: 10.1038/ajh.2010.52 
9. Garrido BJ, Lobera IJ. Interactions between antihypertensive drugs and food. Nutrición Hospitalaria. 2012;27(5):1866-75. doi: 10.3305/ nh.2012.27.6.6127

\section{Laranjeiras R et al. I Levantamento Nacional} sobre os padrões de consumo de álcool na população brasileira. Centro Brasileiro de Informações sobre Drogas Psicotrópicas (CEBRID). Secretaria Nacional Antidrogas (SENAD). Universidade Federal de São Paulo (UNIFESP). Brasília; 2007.

11. Klatsky AL. Alcohol and cardiovascular health. Physiology \& Behavior. 2010;100(1):76-81. doi: 10.1016/i.physbeh.2009.12.019

\section{Klatsky AL, Gunderson E. Alcohol and} hypertension: a review. Journal of the American Society of Hypertension. 2008;2(5):307-17. doi: 10.1016/i.jash.2008.03.010

13. Luo W, Guo Z, Hao C, Yao X, Zhou Z, Wu M et al. Interaction of current alcohol consumption and abdominal obesity on hypertension risk. Physiology \& Behavior. 2013;1 22:182-86. doi: 10.1016/i. physbeh.2012.10.004

14. Simino J, Sung YJ, Kume R, Schwander K, Rao $D C$. Gene alcohol interactions identify several novel blood pressure loci including a promising locus near. Frontiers in Genetics. 2013;4:277. doi: 10.3389/ fgene.2013.00277

\section{Jackson VA, Sesso WD, Buring JE, Gaziano JM.} Alcohol Comsumption and Mortality in Men With Preexisting Cerebrovascular Disease. American Medical Association. 2003; 163: 1 189-93.

\section{Bos S, Grobbee DE, Boer JMA, Verschuren} WM, Beulens JWJ. Alcohol Consumption and Risk of Cardiovascular Disease Among Hypertensive Women. European Journal of Cardiovascular Prevention \& Rehabilitation. 2010;17(1):119-26. doi: $10.1097 /$ HJR.0b013e328335f2fa

17. Halanych JH, Safford MM, Kertesz SG, Pletcher MJ, Kim YI, Person SD et al. Alcohol Consumption in Young Adults and Incident Hypertension: 20Year Follow-up From the Coronary Artery Risk Development in Young Adults Study. American
Journal Epidemiology. 2010;171(5):532-39. doi: $10.1093 / a j e / k w p 417$

18. Wakabayashi I. Relationships among alcohol drinking, blood pressure and serum cholesterol in healthy young women. Clinica Chimica Acta. 2008;388(1-2): 192-195. doi: 10.1016/i. cca.2007.10.013

19. Valencia-Martín JL, Galán I, Guallar-Castillón P, Rodríguez-Artalejo F. Alcohol drinking patterns and health-related quality of life reported in the Spanish adult population. Preventive Medicine. 2013;57(5):703-7. doi: 10.1016/i. ypmed.2013.09.007

20. Pereira RE. Relação entre o consume de bebidas alcoólicas e as infrações de trânsito [Dissertação]. Ribeirão Preto: Faculdade de Ciências Farmacêuticas de Ribeirão Peto/ USP; 2008.

21. Lian C. L'alcoolisme cause d'hypertension artérielle. Bull Acad. Méd. 1915;74:525-28.

22. Steffens AA, Fuchs FD, Moreira LB. Incidência de hipertensão arterial pelo consume de álcool: é modificável pela raça? [Dissertação]. Porto Alegre: Universidade Federal do Rio Grande do Sul. Faculdade de Medicina. Programa de Pós-graduação em Cardiologia e Ciências Cardiovasculares; 2005.

23. Klastsky AL, Friedmann GD, Siegelaub $A B$, Gerard MJ. Alcohol consumption and blood pressure. The New England Journal of Medicine. 1977;296:1 194-2000. doi: 10.1056/ NEJM197705262962103

24. Brasil. Ministério da saúde. Departamento de Atenção Básica. Estratégias Para o Cuidado da Pessoa Com Doença Crônica. Hipertensão Arterial Sistêmica. Brasília. DF. 2013.

25. Reims HM, Kjeldsen SE, Brady WE, Dahlo B, Devereux RB, Julius $S$, et al. Alcohol consumption and cardiovascular risk in hypertensives with left ventricular hypertrophy: the LIFE study. Journal of Human Hypertension. 2004; 18(6):381-89. doi: $10.1038 /$ sj.jhh.1001731 
26. Baynes JW, Dominiczak MH. Bioquímica médica. $2^{a}$ ed. Rio de Janeiro: Elsevier; 2007.

27. Frederico PM. Interações medicamentosas potenciais dos anti-hipertensivos: uso perigoso entre idosos [Dissertação]. Fundação Oswaldo Cruz. Escola Nacional de Saúde Pública Sérgio. Rio de Janeiro: Arouca; 2012.

28. Bibi Z. Retracted. Role of cytochrome P450 in drug interactions. Nutrition \& Metabolism. 2008;5:27. doi: 10.1 186/1743-7075-5-27

29. Goodman \& Gilman. Bases Farmacológicas da terapêutica. $11^{a}$ ed americana. Rio de Janeiro: Artmed; 2006.

30. Morales-Olivas FJ, Estañ L. Antihypertensive drug-drug interactions. Med Clin (Barc). 2005; $124(20): 782-9$.

31. Guimarães S, Moura D, Silva P. Terapêutica Medicamentosa e as suas Bases Farmacológicas. $5^{\text {a }}$ Ed. Porto Editora; 2007.

32. Sikkink, J, Fleming, M. Health effects of alcohol. In Addictive Disorders. (Fleming, MF, and Barry, KL, eds) Mosby - Year Book. ST. Lovis; 1992.

33. Pajak A, Szafraniec K, Kubinova R, Malyutina $S$, Peasey A, Pikhart $H$, et al. Binge Drinking and Blood Pressure: Cross-Sectional Results of the HAPIEE Study. Plos One. 2013;(8)6:e65856. doi: 10.1371 /journal.pone.0065856

34. Arranz S, Chiva-Blanch G, Valderas-Martínez $P$, Medina-Remón A, Lamuela-Raventós RM, Estruch R. Wine, Beer, Alcohol and Polyphenols on Cardiovascular Disease and Cancer. Nutrients. 2012;4(7):759-781. doi: 10.3390/nu4070759

35. Rehm J, Monteiro M. Alcohol consumption and burden of disease in the Americas: implications for alcohol policy. Rev Panam Salud Publica/Pan Am J Public Health. 2005; 1 8(4/5):241-48. doi: $/ 10.1590 / \mathrm{S} 1020-49892005000900003$

\section{Wilsnack RW, Wilsnack SC, Kristjanson AF,} Vogeltanz- Holm ND, Gmel G. Alcohol Consumption: Patterns From The Multinational Genacis Project. National Institutes of Health. 2009;104(9):1487-
37. Stranges $S$, Wu T, Dorn JM, Freudenheim JL, Multi $P$, Farinaro E et al. Relationship of alcohol drinking pattern to risk of hypertension: $a$ population-based study. Jornal of the American Heart Association Hypertension. 2004;44:813-9. doi: $10.1161 / 01$. HYP.0000146537.03103.f2

38. Renaud SC, Gueguen R, Conard P, Lanzmann PD, Orgogozo JM, Henry O. Moderate wine drinkers have lower hypertension-related mortality: a prospective cohort study in French men. The American Journal of Clinical Nutrition. 2004;80(3):62 1-5.

39. Huntgeburth $M$, Freyhaus HT, Rosenkranz S. Alcohol Consumption and Hypertension. Cardiovascular Risk and Hypertension. 2005;7(3):180-85. doi: 10.1007/s1 1906-0050007-2

40. Akhmedionov A, Suvankulov F. Alcohol consumption and its impact on the risk of high blood pressure in Russia. Turkey. Drug and Alcohol Review. 2013;32(3):248-53. doi: $10.1111 /$ i.14653362.2012.00521.x

41. Sesso HD, Cook NR, Buring JE, Manson JE, Gaziano JM. Alcohol Consumption and the Risk of Hypertension in Women and Men. Jornal of the American Heart Association Hypertension. 2008;51:1080-87. doi: 10.1161/ HYPERTENSIONAHA.107.104968

42. Fuchs FD, Chambless LE, Whelton PK, Nieto FJ, Heiss G. Alcohol Consumption and the Incidence of Hypertension: The Atherosclerosis Risk in Communities Study. Journal of the American Heart Association Hypertension. 2001 ; 37:1 242-50. doi: 10.1161/01.HYP.37.5.1242

43. Krauss RM, Eckel RH, Appel IJ, Daniels SR, Deckelbaum RJ, Erdman JW et al. AHA Dietary - Guidelines. A Statement for healthcare professionals from de nutrition Committee of the American Heart Association. Stroke. 2000;31:2751-66.

44. Núnez Córdoba JM, Martínez-González MA, Bes-Rastrollo M, Toledo E, Beunza JJ, Alonso 
A. Alcohol Consumption and the Incidence of Hypertension in a Mediterranean Cohort: The SUN Study. Rev Espanhola de Cardiologia. 2009;62(6):633-41.

45. Ungari $A Q$, Fabbro ALD. Adherence to drug treatment in hypertensive patients on the Family Health Program. Brazilian Journal of Pharmaceutical Sciences. 2010;46(4):81 1-818. doi: $10.1590 /$ S1984-82502010000400024

46. Wang $H$, Wang J, Liu $M M$, Wang $D$, Liu $Y Q$, Zhao $Y$ et al. Epidemiology of general obesity, abdominal obesity and related risk factors in urban adults from 33 communities of northeast china: the CHPSNE study. BMC Public Health. 2012 ; 1 2:967. doi: 10.1186/1471-2458-12-967

47. Silva MEDC, Moura MEB. Representações sociais da hipertensão arterial elaboradas por portadoras e profissionais de saúde: uma contribuição da enfermagem [Dissertação].

Teresina: Universidade Federal do Piauí; 2010.

48. Maciel KF. Mantovani MF. Mazza VA. Rede de apoio social de portadores de hipertensão arterial para o cuidado. [Dissertação Mestrado]. Curitiba; 2010. 